\title{
Duration of antibiotic therapy for bacteremia: a systematic review and meta-analysis
}

Thomas C Havey ${ }^{1}$, Robert A Fowler ${ }^{1,2}$ and Nick Daneman ${ }^{1,3^{*}}$

\begin{abstract}
Introduction: The optimal duration of antibiotic therapy for bloodstream infections is unknown. Shorter durations of therapy have been demonstrated to be as effective as longer durations for many common infections; similar findings in bacteremia could enable hospitals to reduce antibiotic utilization, adverse events, resistance and costs.

Methods: A search of the MEDLINE, EMBASE and COCHRANE databases was conducted for the years 1947-2010. Controlled trials were identified that randomized patients to shorter versus longer durations of treatment for bacteremia, or the infectious foci most commonly causing bacteremia in critically ill patients (catheter-related bloodstream infections (CRBSI), intra-abdominal infections, pneumonia, pyelonephritis and skin and soft-tissue infections (SSTI)).

Results: Twenty-four eligible trials were identified, including one trial focusing exclusively on bacteremia, zero in catheter related bloodstream infection, three in intra-abdominal infection, six in pyelonephritis, thirteen in pneumonia and one in skin and soft tissue infection. Thirteen studies reported on 227 patients with bacteremia allocated to 'shorter' or 'longer' durations of treatment. Outcome data were available for 155 bacteremic patients: neonatal bacteremia ( $n=66$ ); intra-abdominal infection (40); pyelonephritis (9); and pneumonia (40). Among bacteremic patients receiving shorter (5-7 days) versus longer (7-21 days) antibiotic therapy, no significant difference was detected with respect to rates of clinical cure ( $45 / 52$ versus $47 / 49$, risk ratio $0.88,95 \%$ confidence interval [Cl] 0.77-1.01), microbiologic cure (28/28 versus 30/32, risk ratio 1.05, 95\% Cl 0.91-1.21), and survival (15/17 versus $26 / 29$, risk ratio $0.97,95 \% \mathrm{Cl} 0.76-1.23)$.

Conclusions: No significant differences in clinical cure, microbiologic cure and survival were detected among bacteremic patients receiving shorter versus longer duration antibiotic therapy. An adequately powered randomized trial of bacteremic patients is needed to confirm these findings.
\end{abstract}

\section{Introduction}

A recent global point prevalence survey of infections in 1,265 intensive care units (ICUs) documented bloodstream infection (BSI) among $15 \%$ of patients [1], and this rate may be increasing over time because of increased use of immunosuppressive drugs, invasive procedures, and older patients who have concomitant medical conditions and who are admitted to intensive care $[2,3]$. These infections are a major contributor to patient morbidity [1] and are associated with a doubling or even tripling of mortality $[4,5]$. Mortality rates may be higher if delayed [6] or ineffective [7] initial

\footnotetext{
* Correspondence: nick.daneman@sunnybrook.ca

'Department of Medicine, University of Toronto, 1 Kings College Circle, O, M5S 1A8, Canada

Full list of author information is available at the end of the article
}

antimicrobial therapy is prescribed, and so it is difficult to reduce broad-spectrum antibiotic use in the initial empiric phase of treatment in this vulnerable patient population [8]. In contrast, it may be more feasible to reduce antibiotic use at the back end of treatment courses. Up to half of the antibiotic use in hospital wards and critical care units is unnecessary or inappropriate, and excessive durations of treatment are the greatest contributor to inappropriate use [9-11]. A reduction in the length of antibiotic courses is, therefore, a potentially viable strategy to minimize the consequences of antibiotic overuse in critical care, including antibiotic resistance, adverse effects, Clostridium difficile colitis, and costs [12].

The optimal duration of therapy for primary BSI and BSI secondary to major organ system infections has

\section{() Biomed Central}


been poorly defined. A review of the Infectious Diseases Society of America (IDSA) guidelines for the treatment of infections most commonly encountered in the critical care setting - including guidelines for community- and hospital-acquired pneumonia $[13,14]$, intra-abdominal infection [15], catheter-related bloodstream infection (CRBSI) [16], pyelonephritis [17], and skin and soft tissue infection (SSTI) [18] - provides no guidance about the optimal duration of therapy for the subset of patients with bacteremia. In the absence of high-grade evidence, there is wide variability in antibiotic treatment duration recommendations from infectious disease and critical care specialists, and the presence of bacteremia is often used as a justification for extended courses of antibiotic therapy regardless of the observed clinical response to treatment $[19,20]$. Randomized controlled trials (RCTs) examining duration of therapy in several organ system infections have demonstrated that treatment can be shortened to 1 week or less without worsening patient outcomes [11,21-23], so it is plausible that treatment duration could potentially be shortened for BSIs as well.

The objective of this study was to determine whether the therapeutic equivalence of shorter- and longercourse antibiotic therapy extends to patients with bacteremia. We conducted a systematic review and metaanalysis of RCTs explicitly examining the efficacy of shorter-course versus longer-course antibiotic therapy for patients with bacteremia as well as comparable trials examining the organ system infections most commonly causing bacteremia in critically ill patients.

\section{Materials and methods \\ Search strategy}

We searched the Cochrane Central Register of Controlled Trials, Ovid Medline (1948 to 2010), and Ovid Embase (1947 to 2010) to find relevant RCTs comparing shorter versus longer durations of treatment for bacteremia or the five most common organ system infections causing bacteremia in critically ill patients [1]. Filters for RCTs specified by the Scottish Intercollegiate Guidelines Network [24] were combined individually with the following keywords: 'bacteremia', 'bacteraemia', 'pneumonia', 'pyelonephritis', 'cellulitis', 'soft tissue infection', 'skin disease, bacterial', 'peritonitis', 'intra-abdominal infection', 'catheter-related infections', and 'catheterization, central venous AND bacteremia OR bacteraemia OR bloodstream infection'. Results were restricted to trials performed on humans. We manually reviewed the reference lists of retrieved studies, editorials, systematic reviews, and meta-analyses to identify additional relevant trials.

\section{Eligibility criteria}

Clinical trials were included if they used random allocation of patients to treatment groups comparing differing durations of oral, intramuscular, or intravenous antimicrobial treatment of bacteremia, CRBSIs, pneumonia, pyelonephritis, SSTI, or intra-abdominal infections. Eligible trials randomly assigned subjects to one of two different durations of treatment with the same antibiotic regimen and evaluated one or more of the following outcomes: clinical cure, microbiologic cure, or survival. We excluded trials that determined duration of treatment on the basis of physician discretion, clinical improvement, or biomarker measurements. Observational studies were not included, because a large volume of studies was anticipated and because the association of treatment duration and patient outcomes would be impossible to interpret in light of survivor bias and biasby-indication. A sample of 200 citations from each of the six searches (1,200 total citations) was independently reviewed by a second author to assess agreement via calculation of a kappa statistic. Disagreements were resolved through consensus.

\section{Study quality}

The quality of included trials was appraised by using the Cochrane Risk of Bias tool, which assesses sequence generation, allocation concealment, blinding, data completeness, and outcome reporting, and, on the basis of these domains, summarizes studies as exhibiting a low, high, or unclear risk of bias.

\section{Data collection}

Data were collected via a prespecified data extraction spreadsheet with criteria agreed upon by all authors. The information extracted from each trial consisted of the following: (1) infectious syndrome; (2) number of eligible patients screened and randomly assigned; (3) patient characteristics; (4) antibiotic regimen; (5) shorter- and longer-arm treatment duration; (6) day of randomization; (7) allocation sequence method, method of concealment, and presence or absence of blinding strategy; (8) inclusion and exclusion criteria; (9) extent of loss to follow-up; (10) primary outcome measure (including clinical cure, microbiologic cure, and survival); and (11) results of primary outcome in shorterand longer-arm treatment groups and bacteremic subgroups (CRBSI, pneumonia, intra-abdominal infection, pyelonephritis, and SSTI).

\section{Outcomes}

From all trials, we extracted available data on outcomes of clinical cure, microbiologic cure, and survival for the overall groups of patients receiving shorter- and longerduration antibiotic therapy. In our primary analysis, though, we examined these three outcomes (clinical cure, microbiologic cure, and survival) among the subgroup of patients with bacteremia in each treatment 
arm. Therefore, individual studies were closely examined for any information relating to the collection of blood cultures and stated inclusion/exclusion of patients with bacteremia. In studies that included patients with bacteremia, data regarding the proportion with bacteremia in each intervention arm (if available) and outcomes for this bacteremic subgroup (if available) were extracted.

\section{Statistical analysis}

Outcomes with shorter versus longer antibiotic therapy were reported as risk differences and relative risks with 95\% confidence intervals (CIs). In primary analyses, these measures of effect were calculated for the bacteremic subgroups. In secondary analyses, these measures of effect were calculated for the overall study populations. Heterogeneity was assessed across all studies (and for studies within each individual syndrome) via graphical inspection of forest plots as well as calculation of $\mathrm{I}^{2}$ and chi-squared statistics. $P$ values of less than 0.1 were deemed to suggest statistically significant between-study heterogeneity [25]. Pooled relative risks were calculated by using the Mantel-Haenszel fixed effects model. These statistical analyses were performed by using Review Manager version 5 software (The Cochrane Collaboration, Copenhagen, Denmark).

\section{Results}

\section{Study selection and characteristics}

Our search strategy identified 40,484 total references in six separate searches. A total of 24 trials met inclusion criteria, and there was excellent agreement between investigators (kappa statistic $=0.90)$. Only 11 out of 24 studies reported on allocation concealment, 13 out of 24 employed some form of blinding, 10 out of 24 used placebo, and 9 out of 24 had a low risk of bias on the basis of clear reporting of all three of these factors (Table 1). These trials consisted of 1 trial dedicated to bacteremia and 23 trials examining the syndromes most commonly causing critical care-associated bacteremia (0 in CRBSI, 3 in intra-abdominal infection, 6 in pyelonephritis, 1 in SSTI, and 13 in pneumonia) (Figure 1). Fifteen studies (63\%) included patients with bacteremia, and 13 studies (54\%) reported on the proportion of patients with bacteremia ( 1 out of 1 trial in bacteremia, 0 out of 0 in CRBSI, 2 out of 3 in intra-abdominal infection, 2 out of 6 in pyelonephritis, 0 out of 1 in SSTI, and 8 out of 13 in pneumonia).

\section{Outcomes among the overall study populations}

The included studies involved 7,695 participants of varying ages, syndromes, and definitions of shorter and longer therapy (Table 1). Clinical cure (Additional file 1 ), microbiologic cure (Additional file 2), and survival (Additional file 3) were similar for overall study populations receiving shorter-versus longer-duration therapy. Significant heterogeneity across the different infectious syndromes was detected for outcomes of microbiologic cure $\left(\mathrm{I}^{2}=71 \%, P\right.$ value $\left.<0.0001\right)$ but not for clinical cure $\left(\mathrm{I}^{2}=0 \%, P=0.68\right)$ or survival $\left(\mathrm{I}^{2}=0 \%\right.$, $P=0.64)$. Within individual syndromes, there was evidence of between-study heterogeneity for pyelonephritis but not for studies of the other bacteremic syndromes.

\section{Outcomes among patients with bacteremia}

A total of 227 patients with documented bacteremia were described across the included studies (Table 2). Treatment outcomes for patients with bacteremia were available from 7 of these 11 trials (64\%), contributing outcome data for a total of 155 patients with documented positive blood cultures (Table 2). In our primary study analyses, we compared outcomes among bacteremic patients receiving shorter-duration (ranging from 5 to 7 days) versus longer-duration (ranging from 7 to 21 days) antibiotic therapy. Among bacteremic patients receiving shorter versus longer antibiotic therapy, no significant difference was detected with respect to rates of clinical cure $(45 / 52$ versus $47 / 49$, risk ratio $0.88,95 \%$ CI 0.77 to 1.01 ) (Figure 2), microbiologic cure $(28 / 28$ versus $30 / 32$, risk ratio 1.05 , 95\% CI 0.91 to 1.21 ) (Figure 3$)$, or survival ( $15 / 17$ versus $26 / 29$, risk ratio 0.97 , 95\% CI 0.76 to 1.23 ) (Figure 4 ). No significant heterogeneity between studies was detected for clinical cure $\left(\mathrm{I}^{2}=\right.$ $5 \%, P=0.37)$, microbiologic cure $\left(\mathrm{I}^{2}=0 \%, P=0.78\right)$, or survival $\left(\mathrm{I}^{2}=3 \%, P=0.36\right)$.

\section{Detailed outcomes among patients with bacteremia according to underlying infectious syndromes Trials focusing exclusively on bacteremia}

A single randomized trial examined duration of antibiotic therapy exclusively in bacteremia. Chowdhary and colleagues [26] randomly assigned ICU neonates with positive blood cultures and no obvious deep-seated focus of infection to 7 or 14 days of culture-directed antibiotic therapy. Patients were randomly assigned at day 7 if symptoms had clinically remitted by day 5 (32.5\% were excluded because of failure to remit). The composite outcome included bacteriologic relapse with the same organism, a recurrent episode of illness with an elevation in serum C-reactive protein, or a subsequent clinical diagnosis of sepsis by a blinded adjudication committee. No statistically significant difference in treatment outcomes between groups was reported, and 28 out of 33 patients (84.8\%) in the 7 -day arm and 32 out of $33(97 \%)$ in the 14-day arm were successfully treated. In a preplanned subgroup analysis, the success rate for Staphylococcus aureus bacteremia was lower with shorter versus longer therapy: 3 out of 7 (42.9\%) versus 7 out of 7 (100\%), $P=0.02$. Treatment success 
Table 1 Randomized trials of shorter- versus longer-duration antibiotic therapy in bacteremia or syndromes commonly causing bacteremia.

\begin{tabular}{|c|c|c|c|c|c|c|c|c|c|c|}
\hline Author & Syndrome & $\begin{array}{l}\text { Number } \\
\text { screened } \\
\text { (number } \\
\text { randomly } \\
\text { assigned) }\end{array}$ & Population & Antibiotic & $\begin{array}{l}\text { Short } \\
\text { arm, } \\
\text { days }\end{array}$ & $\begin{array}{l}\text { Long } \\
\text { arm, } \\
\text { days }\end{array}$ & $\begin{array}{c}\text { Day of } \\
\text { random } \\
\text { assignment }\end{array}$ & $\begin{array}{c}\text { Random } \\
\text { assignment/ } \\
\text { Concealment }\end{array}$ & $\begin{array}{l}\text { Blinding/ } \\
\text { Placebo }\end{array}$ & $\begin{array}{l}\text { Patients } \\
\text { with } \\
\text { bacteremia }\end{array}$ \\
\hline $\begin{array}{l}\text { Chowdhary } \\
\text { et al. [26] }\end{array}$ & Bacteremia & $229(69)$ & $\begin{array}{l}\text { Hospitalized } \\
\text { neonates }\end{array}$ & Culture-directed & 7 & 14 & 7 & $\begin{array}{c}\text { Computer- } \\
\text { generated/ } \\
\text { Yes }\end{array}$ & Yes/No & Included \\
\hline $\begin{array}{l}\text { Basoli et al. } \\
{[27]}\end{array}$ & Peritonitis & $111(90)$ & $\begin{array}{l}\text { Secondary } \\
\text { peritonitis }\end{array}$ & Ertapenem & 3 & $5-14$ & 3 & $\begin{array}{l}\text { Unclear/ } \\
\text { Unclear }\end{array}$ & Yes/Yes & Unclear \\
\hline $\begin{array}{l}\text { Runyon et } \\
\text { al. [28] }\end{array}$ & Peritonitis & $140(100)$ & $\begin{array}{l}\text { Hospitalized } \\
\text { adults; SBP }\end{array}$ & Cefotaxime & 5 & 10 & 1 & Unclear/No & No/No & Included \\
\hline $\begin{array}{l}\text { Chaudhry et } \\
\text { al. [29] }\end{array}$ & Peritonitis & $60(50)$ & $\begin{array}{l}\text { Hospitalized } \\
\text { adults; SBP }\end{array}$ & Cefoperazone & 5 & 10 & 1 & Unclear/No & No/No & Included \\
\hline $\begin{array}{l}\text { Hepburn et } \\
\text { al. [49] }\end{array}$ & SSTI & $169(87)$ & $\begin{array}{l}\text { Outpatient } \\
\text { adults }\end{array}$ & Levofloxacin & 5 & 10 & 5 & $\begin{array}{c}\text { Computer- } \\
\text { generated/ } \\
\text { Yes }\end{array}$ & Yes/Yes & Excluded \\
\hline $\begin{array}{l}\text { De Gier et } \\
\text { al. [43] }\end{array}$ & Pyelonephritis & N/A (54) & $\begin{array}{l}\text { Hospitalized } \\
\text { adults; } \\
\text { complicated } \\
\text { UTI }\end{array}$ & Fleroxacin & 7 & 14 & 1 & $\begin{array}{l}\text { Unclear/ } \\
\text { Unclear }\end{array}$ & $\begin{array}{l}\text { Unclear/ } \\
\text { No }\end{array}$ & Unclear \\
\hline $\begin{array}{l}\text { Stamm et } \\
\text { al. [44] }\end{array}$ & Pyelonephritis & $98(60)$ & $\begin{array}{l}\text { Outpatient } \\
\text { adult } \\
\text { women }\end{array}$ & $\begin{array}{l}\text { Ampicillin or } \\
\text { TMP/SMX }\end{array}$ & 14 & 42 & 1 & $\begin{array}{l}\text { Random } \\
\text { number } \\
\text { table/No }\end{array}$ & No/No & Unclear \\
\hline $\begin{array}{l}\text { Gleckman } \\
\text { et al. [45] }\end{array}$ & Pyelonephritis & N/A (54) & $\begin{array}{l}\text { Hospitalized } \\
\text { adult } \\
\text { women }\end{array}$ & $\begin{array}{l}\text { Gentamicin/ } \\
\text { tobramycin; } \\
\text { ampicillin, TMP/ } \\
\text { SMX, cephalexin }\end{array}$ & 10 & 21 & 1 & $\begin{array}{l}\text { Random } \\
\text { number } \\
\text { table/No }\end{array}$ & No/No & Unclear \\
\hline $\begin{array}{l}\text { Pylkkanen } \\
\text { et al. [46] }\end{array}$ & Pyelonephritis & 271 (149) & $\begin{array}{l}\text { Infants and } \\
\text { children }\end{array}$ & Sulfafurazole & 10 & 42 & 1 & Unclear/No & No/No & Unclear \\
\hline $\begin{array}{l}\text { Jernelius et } \\
\text { al. [47] }\end{array}$ & Pyelonephritis & N/A (77) & $\begin{array}{l}\text { Outpatient } \\
\text { and } \\
\text { hospitalized } \\
\text { adults }\end{array}$ & $\begin{array}{l}\text { Pivampicillin } \\
\text { and } \\
\text { pivmecillinam }\end{array}$ & 7 & 21 & 1 & $\begin{array}{l}\text { Computer- } \\
\text { generated/ } \\
\text { Yes }\end{array}$ & Yes/Yes & Included \\
\hline $\begin{array}{l}\text { Cheng et al. } \\
\text { [48] }\end{array}$ & Pyelonephritis & N/A (80) & $\begin{array}{l}\text { Hospitalized } \\
\text { children }\end{array}$ & Acc. to culture & 14 & 21 & 1 & $\begin{array}{l}\text { Serial entry/ } \\
\text { Unclear }\end{array}$ & $\begin{array}{c}\text { Unclear/ } \\
\text { No }\end{array}$ & Included \\
\hline $\begin{array}{l}\text { Engle et al. } \\
{[30]}\end{array}$ & Pneumonia & $51(26)$ & $\begin{array}{l}\text { Hospitalized } \\
\text { neonates }\end{array}$ & $\begin{array}{l}\text { Ampicillin and } \\
\text { gentamicin }\end{array}$ & 2 & 4 & 2 & $\begin{array}{l}\text { Unclear/ } \\
\text { Unclear }\end{array}$ & $\begin{array}{l}\text { Unclear/ } \\
\text { No }\end{array}$ & Included \\
\hline $\begin{array}{l}\text { Engle et al. } \\
\text { [31] }\end{array}$ & Pneumonia & $212(73)$ & $\begin{array}{l}\text { Hospitalized } \\
\text { neonates }\end{array}$ & $\begin{array}{l}\text { Ampicillin and } \\
\text { gentamicin }\end{array}$ & 4 & 7 & 2 & Unclear/Yes & $\begin{array}{c}\text { Unclear/ } \\
\text { No }\end{array}$ & Included \\
\hline ISCAP [33] & Pneumonia & N/A $(2,188)$ & $\begin{array}{l}\text { Outpatient } \\
\text { children } 2 \text { to } \\
59 \text { months }\end{array}$ & Amoxicillin & 3 & 5 & 1 & Unclear/Yes & Yes/Yes & Unclear \\
\hline $\begin{array}{l}\text { MASCOT } \\
{[34]}\end{array}$ & Pneumonia & N/A $(2,000)$ & $\begin{array}{l}\text { Outpatient } \\
\text { children } 2 \text { to } \\
59 \text { months }\end{array}$ & Amoxicillin & 3 & 5 & 1 & $\begin{array}{l}\text { Computer- } \\
\text { generated/ } \\
\text { Yes }\end{array}$ & Yes/Yes & Unclear \\
\hline $\begin{array}{l}\text { Vuori- } \\
\text { Holopainen } \\
\text { et al. [32] }\end{array}$ & Pneumonia & $178(72)$ & $\begin{array}{l}\text { Hospitalized } \\
\text { children } 3 \\
\text { months to } \\
15 \text { years }\end{array}$ & $\begin{array}{l}\text { Penicillin or } \\
\text { cefuroxime }\end{array}$ & 4 & 7 & 1 & $\begin{array}{l}\text { Computer- } \\
\text { generated/No }\end{array}$ & $\mathrm{No} / \mathrm{No}$ & Included \\
\hline $\begin{array}{l}\text { Tellier et al. } \\
{[36]}\end{array}$ & Pneumonia & $581(575)$ & $\begin{array}{l}\text { Outpatient } \\
\text { and } \\
\text { hospitalized } \\
\text { adults }\end{array}$ & Telithromycin & 5 & 7 & 1 & Unclear/Yes & Yes/Yes & Included \\
\hline $\begin{array}{l}\text { File et al. } \\
{[35]}\end{array}$ & Pneumonia & N/A (512) & $\begin{array}{l}\text { Outpatient } \\
\text { adults }\end{array}$ & Gemifloxacin & 5 & 7 & 1 & $\begin{array}{l}\text { Unclear/ } \\
\text { Unclear }\end{array}$ & $\begin{array}{l}\text { Yes/ } \\
\text { Unclear }\end{array}$ & Included \\
\hline $\begin{array}{l}\text { Dunbar et } \\
\text { al. [37] }\end{array}$ & Pneumonia & $2,521(530)$ & $\begin{array}{l}\text { Outpatient } \\
\text { and } \\
\text { hospitalized } \\
\text { adults }\end{array}$ & Levofloxacin & 5 & 10 & 1 & Unclear/Yes & Yes/Yes & Included \\
\hline
\end{tabular}


Table 1 Randomized trials of shorter- versus longer-duration antibiotic therapy in bacteremia or syndromes commonly causing bacteremia. (Continued)

\begin{tabular}{|c|c|c|c|c|c|c|c|c|c|c|}
\hline $\begin{array}{l}\text { Leophonte } \\
\text { et al. [39] }\end{array}$ & Pneumonia & N/A (244) & $\begin{array}{l}\text { Hospitalized } \\
\text { adults }\end{array}$ & Ceftriaxone & 5 & 10 & 1 & Unclear/Yes & Yes/Yes & Included \\
\hline $\begin{array}{l}\text { Siegel et al. } \\
\text { [38] }\end{array}$ & Pneumonia & N/A (52) & $\begin{array}{l}\text { Hospitalized } \\
\text { adults }\end{array}$ & Cefuroxime & 7 & 10 & 1 & $\begin{array}{c}\text { Computer- } \\
\text { generated/ } \\
\text { Yes }\end{array}$ & Yes/Yes & Included \\
\hline $\begin{array}{l}\text { El } \\
\text { Moussaoui } \\
\text { et al. [40] }\end{array}$ & Pneumonia & $186(121)$ & $\begin{array}{l}\text { Hospitalized } \\
\text { adults }\end{array}$ & Amoxicillin & 3 & 8 & 3 & Unclear/Yes & Yes/Yes & Included \\
\hline $\begin{array}{l}\text { Chastre et } \\
\text { al. [42] }\end{array}$ & Pneumonia & $1,171(402)$ & $\begin{array}{l}\text { Hospitalized } \\
\text { adults }\end{array}$ & Culture-directed & 8 & 15 & 3 & $\begin{array}{c}\text { Computer- } \\
\text { generated/ } \\
\text { Yes }\end{array}$ & Yes/No & Included \\
\hline $\begin{array}{l}\text { Fekih } \\
\text { Hassen et } \\
\text { al. [41] }\end{array}$ & Pneumonia & $39(30)$ & $\begin{array}{l}\text { Hospitalized } \\
\text { adults }\end{array}$ & Culture-directed & 7 & 10 & 2 & $\begin{array}{l}\text { Random } \\
\text { number } \\
\text { table/No }\end{array}$ & No/No & Unclear \\
\hline
\end{tabular}

ISCAP, IndiaClen Short Course Amoxicillin Pneumonia; MASCOT, Multicentre Amoxicillin Short Course Therapy; N/A, not applicable; SBP, spontaneous bacterial peritonitis; SSTI, skin and soft tissue infection; TMP/SMX, trimethoprim/sulfamethoxazole; UTI, urinary tract infection.

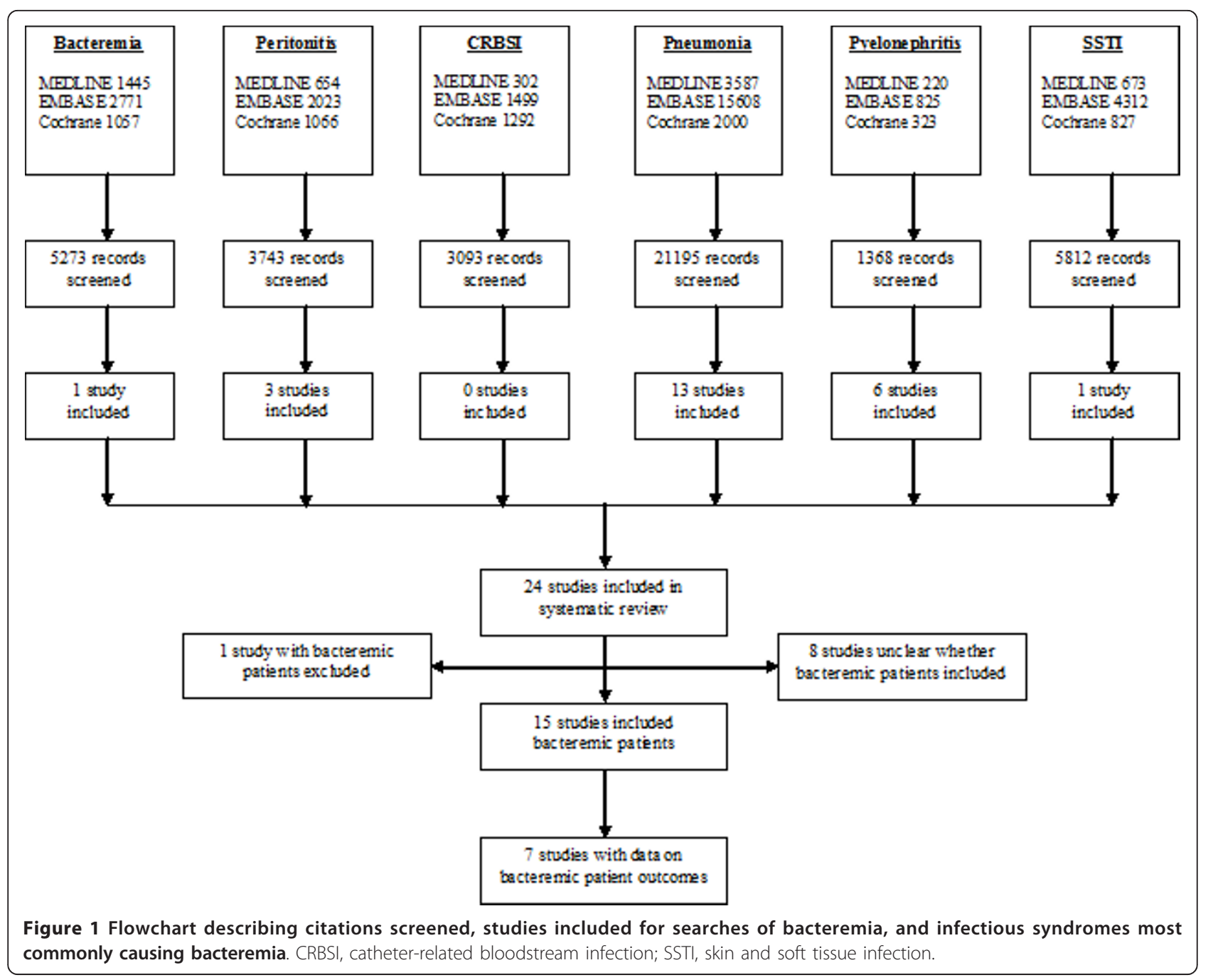


Table 2 Randomized controlled trials of shorter versus longer antibiotic therapy that included patients with bacteremia.

\begin{tabular}{|c|c|c|c|c|}
\hline Author & Syndrome & $\begin{array}{l}\text { All patients blood } \\
\text { cultured? }\end{array}$ & $\begin{array}{l}\text { Short-arm group, number } \\
\text { (percentage) bacteremic }\end{array}$ & $\begin{array}{c}\text { Long-arm group, number } \\
\text { (percentage) bacteremic }\end{array}$ \\
\hline \multirow[t]{2}{*}{$\begin{array}{l}\text { Chowdhary et al. } \\
\text { [26] }\end{array}$} & $\begin{array}{c}\text { Non-Staphylococcus aureus } \\
\text { bacteremia }\end{array}$ & Yes & $26 / 26(100 \%)$ & $26 / 26(100 \%)$ \\
\hline & S. aureus bacteremia & Yes & $7 / 7(100 \%)$ & $7 / 7(100 \%)$ \\
\hline Runyon et al. [28] & Peritonitis & Yes & 9/43 (20.9\%) & $17 / 47(36.2 \%)$ \\
\hline $\begin{array}{l}\text { Chaudhry et al. } \\
\text { [29] }\end{array}$ & Peritonitis & Yes & $6 / 25(24 \%)$ & $8 / 25(32 \%)$ \\
\hline Jernelius et al. [47] & Pyelonephritis & Yes & $5 / 32(15.6 \%)$ & $4 / 29(13.8 \%)$ \\
\hline Cheng et al. [48] & Pyelonephritis & Yes & $1 / 41(2.4 \%)$ & $3 / 39(7.7 \%)$ \\
\hline Engle et al. [30] & Pneumonia & Yes & 0/14 (0\%) & 0/12 (0\%) \\
\hline Engle et al. [31] & Pneumonia & Yes & $0 / 35(0 \%)$ & 0/38 (0\%) \\
\hline $\begin{array}{l}\text { Vuori-Holopainen } \\
\text { et al. [32] }\end{array}$ & Pneumonia & Yes & N/A & N/A \\
\hline Tellier et al. [36] & Pneumonia & Yes & 14/187 (7.5\%) & 9/191 (4.7\%) \\
\hline File et al. [35] & Pneumonia & Yes & N/A & N/A \\
\hline Dunbar et al. [37] & Pneumonia & Yes & $7 / 256(2.7 \%)$ & $7 / 272$ (2.6\%) \\
\hline $\begin{array}{l}\text { Leophonte et al. } \\
\text { [39] }\end{array}$ & Pneumonia & Yes & $11 / 125(8.8 \%)$ & $12 / 119(10.1 \%)$ \\
\hline Siegel et al. [38] & Pneumonia & Yes & $2 / 24(8.3 \%)$ & $4 / 22(18.2 \%)$ \\
\hline $\begin{array}{l}\text { El Moussaoui et al. } \\
{[40]}\end{array}$ & Pneumonia & Unclear & $6 / 56(10.7 \%)$ & $8 / 63(12.7 \%)$ \\
\hline Chastre et al. [42] & Pneumonia & Yes & 14/197 (7.1\%) & $14 / 204$ (6.9\%) \\
\hline
\end{tabular}

N/A, not applicable.

was not significantly different for shorter and longer duration of therapy in non-S. aureus infections (25 out of 26 in both groups).

\section{Catheter-related bloodstream infection}

A search of 3,093 indexed citations from Medline, Embase, and the Cochrane Database did not yield any eligible randomized trials examining duration of antibiotic therapy in CRBSI.

\section{Intra-abdominal infection}

Three randomized trials examined duration of antibiotic therapy in intra-abdominal infection. The first randomly assigned 90 adult patients with community-acquired localized secondary intra-abdominal infections of mild to moderate severity to 3 or at least 5 days (mean of 5.7 days and range of 5 to 10 days) of postoperative ertapenem [27]. Patients were included if symptoms and leukocytosis had improved by day 3. Clinical cure was achieved in 39 out of 42 patients $(92.9 \%)$ receiving shorter therapy and in 43 out of 48 (89.6\%) receiving longer therapy. Although blood cultures were drawn when 'clinically indicated', the prevalence of bacteremia and outcomes in patients with bacteremia were not reported [27].

Two unblinded randomized trials [28,29] compared 5 versus 10 days of cephalosporin therapy in patients with spontaneous bacterial peritonitis (SBP). All patients underwent blood cultures at enrollment. In one trial, 26 out of $90(28.9 \%)$ were bacteremic, and no significant difference in survival was observed for shorter (43 out

\begin{tabular}{|c|c|c|c|c|c|c|c|}
\hline Study or Subgroup & \multicolumn{2}{|c|}{ Short Duratlon } & \multicolumn{2}{|c|}{ Long Duration } & Welght & $\begin{array}{l}\text { Rlsk Ratlo } \\
\text { M-H, Flxed, 95\% Cl }\end{array}$ & $\begin{array}{c}\text { RIsk Ratlo } \\
\text { MH, Flxed, } 95 \% \text { Cl }\end{array}$ \\
\hline Chowdhary & 28 & 33 & 32 & 33 & $64.5 \%$ & $0.88[0.75,1.02]$ & \\
\hline Jernelius & 5 & 5 & 4 & 4 & $9.9 \%$ & $1.00[0.68,1.46]$ & $\longrightarrow$ \\
\hline Siegel & 0 & 2 & 3 & 4 & $5.3 \%$ & $0.24[0.02,3.19]$ & \\
\hline Tellier & 12 & 12 & 8 & 8 & $20.3 \%$ & $1.00[0.83,1.21]$ & + \\
\hline Total $(95 \% \mathrm{Cl})$ & & 52 & & 49 & $100.0 \%$ & $0.88[0.77,1.01]$ & A \\
\hline Total events & 45 & & 47 & & & & \\
\hline $\begin{array}{l}\text { Heterogeneity: } \mathrm{Chi}^{2}= \\
\text { Test for overall effect: }\end{array}$ & $\begin{array}{l}3.15, d f=3 \\
Z=1.86(P\end{array}$ & $\begin{array}{l}P=0.3 \\
=0.06)\end{array}$ & 7); $1^{2}=5 \%$ & & & & 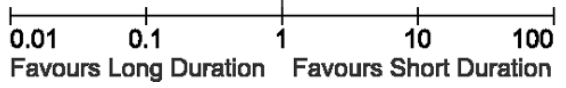 \\
\hline
\end{tabular}




\begin{tabular}{|c|c|c|c|c|c|c|c|}
\hline Study or Subgroup & $\begin{array}{l}\text { Short Du } \\
\text { Events }\end{array}$ & $\begin{array}{l}\text { atlon } \\
\text { Total }\end{array}$ & $\begin{array}{l}\text { Long Dur } \\
\text { Events }\end{array}$ & $\begin{array}{l}\text { atlon } \\
\text { Total }\end{array}$ & Weight & $\begin{array}{l}\text { Rlsk Ratlo } \\
\text { M-H, Fixed, } 95 \% \text { CI }\end{array}$ & $\begin{array}{l}\text { RIsk Ratlo } \\
\text { MH, Fixed, } 95 \% \text { CI }\end{array}$ \\
\hline Dunbar & 7 & 7 & 6 & 7 & $22.9 \%$ & $1.15[0.79,1.68]$ & \\
\hline Runyon & 9 & 9 & 16 & 17 & $41.6 \%$ & $1.04[0.85,1.26]$ & \\
\hline Tellier & 12 & 12 & 8 & 8 & $35.5 \%$ & $1.00[0.83,1.21]$ & \\
\hline Total $(95 \% \mathrm{Cl})$ & & 28 & & 32 & $100.0 \%$ & $1.05[0.91,1.21]$ & 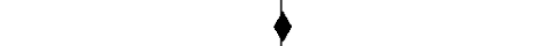 \\
\hline Total events & 28 & & 30 & & & & \\
\hline \multicolumn{7}{|c|}{$\begin{array}{l}\text { Heterogeneity: } \mathrm{Ch}^{2}=0.51, \mathrm{df}=2(\mathrm{P}=0.78) ; \mathrm{l}^{2}=0 \% \\
\text { Test for overall effect: } Z=0.69(P=0.49)\end{array}$} & 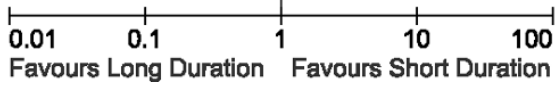 \\
\hline
\end{tabular}

Figure 3 Forest plot for outcome of microbiologic cure among bacteremic subgroups of randomized trials of shorter versus longer antibiotic treatment. $\mathrm{Cl}$, confidence interval; $\mathrm{df}$, degrees of freedom; $\mathrm{M}-\mathrm{H}$, Mantel-Haenszel.

of 43) versus longer (45 out of 47) treatment [28]. Infection-related mortality among bacteremic patients treated for 5 days $(0 \%)$ and 10 days $(11.8 \%)$ was not significantly different [28]. In the second trial, 14 out of $50(28 \%)$ were bacteremic, and no significant difference in outcome was observed; $83.3 \%$ of short-arm and $87.5 \%$ of long-arm patients survived their infections [29]. Secondary outcomes of relapse and hospitalization mortality were also similar between treatment arms and for bacteremic subgroups [28,29].

\section{Pneumonia}

Thirteen eligible trials that included a total of 6,825 patients of various ages, clinical settings, and treatments were included (Table 1). Five trials examined treatment duration in pediatric pneumonia, six studied adult community-acquired pneumonia (CAP), and two studied ventilator-associated pneumonia (VAP).

Clinical success rates were similar with shorter versus longer therapy in five studies of neonatal pneumonia (Table 1 and Figure 2) [30-34]. These studies either documented no patients with bacteremia $[30,31]$ or did not report on this subgroup [32-34].

Six trials of adult CAP found no difference in clinical effectiveness of shorter versus longer duration of treatment [35-40]. Five trials included information on patients with bacteremia [36-40], and three provided subgroup analyses of outcomes of patients with bacteremia [36-38]. In a trial of mild to moderate CAP, 388 patients were randomly assigned to 5 or 7 days of treatment with telithromycin [36]. Blood cultures were positive in 23 out of 388 (5.2\%), and treatment was successful for 20 out of 20 patients with Streptococcus pneumoniae bacteremia. The pathogens implicated in the remaining three bacteremias (and the corresponding patient outcomes) were not reported. In a trial of 530 patients with mild to moderate CAP [37], only 14 (2.7\%) were bacteremic, and no significant difference in clinical success was noted between 7 out of 7 bacteremic patients receiving 5 days of levofloxacin and 6 out of 7 (85.7\%) receiving 10 days. Finally, a trial $(n=52)$ of adult inpatients with CAP documented bacteremia in 6 out of 46 patients (13\%). Neither of 2 bacteremic patients receiving 7 days of cefuroxime achieved clinical success (although 1 died within 24 hours of initiation) versus 3 out of 4 patients receiving 10 days [38].

One small VAP trial randomly assigned 30 patients to 7 or 10 days of culture-directed antibiotic therapy, and similar rates of survival among those treated for 7 days $(64.3 \%)$ and 10 days (62.5\%) were reported [41]. The numbers of patients with bacteremia or their outcomes were not provided [41]. In a large, multicenter, highquality trial that randomly assigned 402 adult patients to 8 or 15 days of therapy [42], survival was not different: 160 out of 197 (81.2\%) versus 169 out of 204 (82.8\%) [42]. While all patients were blood cultured at enrollment and 28 out of 402 patients (7\%) were

\begin{tabular}{|c|c|c|c|c|c|c|c|}
\hline Study or Subgroup & $\begin{array}{l}\text { Short Dut } \\
\text { Events }\end{array}$ & $\begin{array}{l}\text { atlon } \\
\text { Total }\end{array}$ & $\begin{array}{l}\text { Long Dur } \\
\text { Events }\end{array}$ & $\begin{array}{l}\text { ation } \\
\text { Total }\end{array}$ & Weight & $\begin{array}{l}\text { RIsk Ratlo } \\
\text { M-H, Fixed, 95\% Cl }\end{array}$ & $\begin{array}{c}\text { RIsk Ratlo } \\
\text { M-H, Fixed, 95\% Cl }\end{array}$ \\
\hline Chaudhry & 5 & 6 & 7 & 8 & $29.3 \%$ & $0.95[0.61,1.48]$ & $\rightarrow$ \\
\hline Runyon & 9 & 9 & 15 & 17 & $54.1 \%$ & $1.10[0.87,1.39]$ & \\
\hline Siegel & 1 & 2 & 4 & 4 & $16.5 \%$ & $0.56[0.17,1.79]$ & \\
\hline Total $(95 \% \mathrm{Cl})$ & & 17 & & 29 & $100.0 \%$ & $0.97[0.76,1.23]$ & \\
\hline Total events & 15 & & 26 & & & & \\
\hline \multicolumn{7}{|c|}{$\begin{array}{l}\text { Heterogeneity: } \mathrm{Chi}^{2}=2.07, \mathrm{df}=2(P=0.36) ; \mathrm{I}^{2}=3 \% \\
\text { Test for overall effect: } Z=0.26(P=0.79)\end{array}$} & \begin{tabular}{|ccccc}
0.01 & 0.1 & 1 & 10 & 100 \\
Favours Long Duration & Favours Short Duration
\end{tabular} \\
\hline
\end{tabular}


bacteremic, the outcomes of these patients with bacteremia were not separately reported [42].

\section{Pyelonephritis}

Six trials of treatment duration for pyelonephritis were included [43-48]. Only two out of six trials reported outcomes for patients with bacteremia $[47,48]$. In a blinded, placebo-controlled trial, 77 adults with pyelonephritis were randomly assigned to 7 versus 21 days of treatment with pivampicillin and pivmecillinam [47]. All patients underwent blood cultures at enrollment, and 9 out of $61(14.8 \%)$ were bacteremic [47]. Clinical cure was achieved in 4 out of 4 bacteremic patients receiving 7 days and 5 out of 5 bacteremic patients receiving 21 days of treatment [47]. A second trial involved 14 versus 21 days of antibiotics for 80 children with acute lobar nephronia, defined as an advanced state of pyelonephritis which may progress to renal abscess if left untreated [48]. Clinical cure was lower among patients receiving shorter versus longer therapy (34 out of 41 versus 39 out of $39, P=0.01$ ) [48]. Four patients were bacteremic, but their outcomes were not provided [48].

\section{Skin and soft tissue infection}

A single randomized trial of antibiotic duration in SSTI was identified [49]. Adult patients with uncomplicated SSTIs were randomly assigned to 5 or 10 days of therapy with levofloxacin but only if they were improving after 5 days of treatment [49]. Clinical cure was observed in 43 out of 44 patients $(97.7 \%)$ receiving 5 days of therapy and in 42 out of 43 (97.7\%) receiving 10 days [49]. Blood cultures were drawn from eight patients when bacteremia was suspected and were positive in one patient, who was excluded from the trial [49].

\section{Discussion}

This systematic review and meta-analysis identified only one RCT examining shorter versus longer duration of antibiotic therapy exclusively for patients with bacteremia and a further 23 trials among patients with the infectious syndromes that are most commonly associated with bacteremia in the ICU. No significant difference in cure or survival was detected for bacteremic patients receiving shorter- versus longer-duration therapy. These data indicate that shorter-duration therapy may be effective for BSIs but also highlight the need for a larger, high-quality trial dedicated to this question.

The only trial randomly assigning exclusively patients with bacteremia, rather than an identified infectious syndrome, was conducted in a severely ill neonatal population [26]. The main finding of this trial was that overall outcomes were not significantly different for neonates receiving shorter- versus longer-duration antibiotics. A high rate of failure was seen among the small number of patients receiving short-duration treatment for $S$. aureus bacteremia, and this is consistent with the findings of some prior retrospective studies and current treatment guidelines for this pathogen [50,51]; yet other studies support short-course therapy for catheter-associated $S$. aureus bacteremia [52]. This review highlights the potential importance of considering $S$. aureus bacteremia separately from other pathogens in the context of adequately powered trials in the future.

Although bacteremia caused by intravascular catheters is often cited as the commonest cause of BSI in the critical care setting, we were unable to identify any prospective, randomized investigations examining the duration of antibiotic therapy for CRBSI. Recommendations for length of treatment from the current IDSA guidelines are based only on expert opinion and retrospective case series and call for 5 to 7 days for infections caused by coagulase-negative staphylococci, 7 to 14 days for Enterococci and Gram-negative organisms, and 2 to 6 weeks for $S$. aureus [16]. Trials are urgently needed, as this may be the syndrome most appropriate for shorter-course therapy given that the focus of infection is removable and thereby leaves no persisting infectious nidus for most patients.

The available trials randomly assigning patients with intra-abdominal infections to shorter versus longer durations of antibiotic therapy were conducted in nonICU settings and populations and explicitly excluded those with generalized secondary peritonitis or nosocomial infections. Equivalent outcomes for shorter- versus longer-duration therapy for SBP and localized intraabdominal infection are in keeping with similar findings in a retrospective analysis of 929 patients with intraabdominal infections, in which less than 7 days of therapy was not associated with higher complications or mortality [53]. We have not uncovered evidence, though, of whether the effectiveness of short-duration therapy extends to patients with severe infections complicated by bacteremia or in those for whom source control cannot readily be achieved.

Several narrative reviews [54,55] and meta-analyses $[22,23]$ of RCTs have provided evidence of the efficacy and safety of treating mild to moderate CAP with shortduration antibiotic therapy (5 to 7 days). The 13 studies of pneumonia identified for this review included 5 studies of CAP that provided the prevalence of bacteremia; in total, 80 patients with bacteremia were randomly assigned in equal numbers to short (3 to 7 days) and long (7 to 10 days) durations of therapy. Only three studies provided outcome data for bacteremic subgroups, and clinical cure was reported in 19 out of 21 patients (89.4\%) receiving short-duration therapy (5 to 7 days) and in 17 out of 19 patients $(90.4 \%)$ receiving extended therapy ( 7 to 10 days). These results provide a modicum of support for the growing consensus that CAP may be safely treated with shorter durations of therapy 
irrespective of the presence of bacteremia [11,12]. Although a large VAP trial documented equivalent survival with shorter (8 days) versus longer (15 days) treatment [42], very few of these patients were bacteremic, and it is unclear whether shorter-duration treatment can be extended to this subgroup.

The eligible trials of treatment duration in pyelonephritis involved diverse patient populations, durations of treatment, and outcome measures, and this explains the heterogeneity of outcomes with shorter durations of therapy. Recently, the results of several RCTs have demonstrated short-course (5 or 7 days) fluoroquinolone therapy to be equally as efficacious as 10 to 14 days of treatment with comparator medications [56-58]. IDSA guidelines were accordingly amended to recommend therapy for 7 days [17], but the issue of bacteremia was not explicitly addressed. Our data suggest that patients with bacteremia secondary to uncomplicated pyelonephritis can be successfully treated with shorter-duration therapy.

The sole trial of treatment duration in SSTI demonstrated that, in a carefully selected healthy adult population, a short duration of therapy was associated with a cure rate equal to that of a long duration of therapy [49]. However, the explicit exclusion of patients with more serious infections, argues that it is unlikely that these data can be directly extrapolated to critically ill patients with bacteremic soft tissue infections.

The present review has several important limitations. The prespecified search strategy excluded unpublished data and non-English language trials. Of eligible trials, bias may have been introduced by low rates of blinding and use of placebo controls. Some studies included only patients with early clinical improvement for randomization to short- or long-course therapy; consequently, the effect size and findings may not be generalizable to sicker patient populations. Other trials excluded patients post-randomization or presented only per-protocol analyses and so may have excluded bacteremic patients failing therapy. Treatment outcomes for patients with bacteremia in individual studies were derived from small post hoc subgroup analyses. Finally, within individual infectious syndromes, significant variability was encountered both in study design and in the durations of therapy employed (with 14 days even considered shortercourse therapy in one study). However, the lack of outcome heterogeneity between syndromes suggests that it is valid to pool BSI data from multiple infectious foci into a single meta-analysis or enroll such patients within a single RCT.

\section{Conclusions}

The ICU is the epicenter of bacteremia, antibiotic use, and antibiotic resistance in most hospitals. Reductions in the length of antibiotic treatment courses could potentially limit antibiotic use, adverse effects, and resistance pressure, but antibiotic stewardship efforts to shorten therapy are hampered by the lack of research regarding minimally acceptable durations of treatment for BSIs. Our systematic review and meta-analysis indicate that both inpatients and outpatients with non-S. aureus bacteremia secondary to mild to moderate intraabdominal infection, CAP, or pyelonephritis may be successfully treated with shorter (5 to 7 days) courses of therapy. However, this finding must be interpreted with caution, as only very small numbers of patients and subgroup analyses are currently available for interpretation. A large dedicated trial of treatment duration for bacteremia in severely ill patients is urgently needed to determine the optimal duration of therapy.

\section{Key messages}

- The optimal duration of treatment for bloodstream infections is understudied.

- Available data from bacteremic subgroups of prior randomized controlled trials suggest that shorterduration therapy (not more than 7 days) may be as effective as longer-duration therapy in achieving clinical cure, microbiologic cure, and survival among most patients with bloodstream infections.

- A large dedicated randomized trial of treatment duration for bacteremia is urgently needed.

\section{Additional material}

Additional file 1: Forest plot for outcome of clinical cure among overall study populations (irrespective of presence or absence of bacteremia) in trials of bacteremia and each of the most common infectious syndromes causing bacteremia (SSTI = skin and soft tissue infection)

Additional file 2: Forest plot for outcome of microbiologic cure among overall study populations (irrespective of presence or absence of bacteremia) in trials of bacteremia and each of the most common infectious syndromes causing bacteremia

Additional file 3: Forest plot for outcome of survival among overall study populations (irrespective of presence or absence of bacteremia) in trials of bacteremia and each of the most common infectious syndromes causing bacteremia

\section{Abbreviations}

BSI: bloodstream infection; CAP: community-acquired pneumonia; $\mathrm{Cl}$ : confidence interval; CRBSI: catheter-related bloodstream infection; ICU: intensive care unit; IDSA: Infectious Diseases Society of America; RCT: randomized controlled trial; SBP: spontaneous bacterial peritonitis; SSTI: skin and soft tissue infection; VAP: ventilator-associated pneumonia.

\section{Acknowledgements}

ND is supported by a Clinician Scientist Award from the Canadian Institutes of Health Research. RAF is a Clinician Scientist of the Heart and Stroke

Foundation. Additional funding for this study was provided by an Ontario Ministry of Health and Long-term Care Academic Health Sciences Centre Innovation Fund Award. The funding bodies had no role in study design, 
data collection or interpretation, manuscript writing, or the decision to submit the manuscript for publication.

\section{Author details}

'Department of Medicine, University of Toronto, 1 Kings College Circle, O, M5S 1A8, Canada. ${ }^{2}$ Department of Critical Care Medicine, Sunnybrook Health Sciences Centre, 2075 Bayview Avenue, University of Toronto, M4N 3N5 Canada. ${ }^{3}$ Division of Infectious Diseases, Sunnybrook Health Sciences Centre, 2075 Bayview Avenue, University of Toronto, M4N 3N5 Canada.

\section{Authors' contributions}

All authors contributed to the inception of the research question and study design. TCH executed the main database searches and helped to extract data from individual studies by using prespecified methods determined by all study authors. ND independently reviewed a subset of 1,200 citations and helped to extract data from individual studies by using prespecified methods determined by all study authors. All authors contributed to drafting the manuscript and read and approved the final manuscript.

\section{Competing interests}

The authors declare that they have no competing interests.

Received: 7 September 2011 Revised: 8 September 2011

Accepted: 15 November 2011 Published: 15 November 2011

\section{References}

1. Vincent JL, Rello J, Marshall J, Silva E, Anzueto A, Martin CD, Moreno R, Lipman J, Gomersall C, Sakr Y, Reinhart K, EPIC II Group of Investigators: International study of the prevalence and outcomes of infection in intensive care units. JAMA 2009, 302:2323-2329.

2. Valles J, Leon C, Alvarez-Lerma F: Nosocomial bacteremia in critically ill patients: a multicenter study evaluating epidemiology and prognosis. Clin Infect Dis 1997, 24:387-395.

3. Weinstein MP, Towns ML, Quartey SM, Mirrett S, Reimer LG, Parmigiani G, Reller LB: The clinical significance of positive blood cultures in the 1990s: a prospective comprehensive evaluation of the microbiology, epidemiology, and outcome of bacteremia and fungemia in adults. Clin Infect Dis 1997, 24:584-602.

4. Renaud B, Brun-Buisson C: Outcomes of primary and catheter-related bacteremia. Am J Respir Crit Care Med 2001, 163:1584-1590.

5. Garrouste-Orgeas M, Timsit JF, Tafflet M, Misset B, Zahar JR, Soufir L, Lazard T, Jamali S, Mourvillier B, Cohen Y, De Lassence A, Azoulay E, Cheval C, Descorps-Declere A, Adrie C, Costa de Beauregard MA, Carlet J, OUTCOMEREA Study Group: Excess risk of death from intensive care unitacquired nosocomial bloodstream infections: a reappraisal. Clin Infect Dis 2006, 42:1118-1126.

6. Kumar A, Roberts D, Wood KE, Light B, Parrillo JE, Sharma S, Suppes R, Feinstein D, Zanotti S, Taiberg L, Gurka D, Kumar A, Cheang M: Duration of hypotension before initiation of effective antimicrobial therapy is the critical determinant of survival in human septic shock. Crit Care Med 2006, 34:1589-1596.

7. Ibrahim EH, Sherman G, Ward S, Fraser VJ, Kollef MH: The influence of inadequate antimicrobial treatment on patient outcomes in the ICU setting. Chest 2000, 118:146-155.

8. McGregor JC, Rich SE, Harris AD, Perencevich EN, Osih R, Lodise TP Jr, Miller RR, Furuno JP: A systematic review of the methods used to assess the association between appropriate antibiotic therapy and mortality in bacteremic patients. Clin Infect Dis 2007, 45:329-337.

9. Hecker MT, Aron DC, Patel NP, Lehmann MK, Donskey CJ: Unnecessary use of antimicrobials in hospitalized patients. Arch Intern Med 2003, 163:972-978.

10. Rice LB: The Maxwell Finland lecture: for the duration-rational antibiotic administration in an era of antimicrobial resistance and Clostridium difficile. Clin Infect Dis 2008, 46:491-496.

11. Hayashi $Y$, Paterson DL: Strategies for reduction in duration of antibiotic use in hospitalized patients. Clin Infect Dis 2011, 52:1232-1240.

12. Rubinstein E: Short antibiotic treatment courses or how short is short? Int J Antimicrob Agents 2007, 30:76-79.

13. Mandell LA, Wunderink RG, Anzueto A, Bartlett JG, Campbell GD, Dean NC, Dowell SF, File TM Jr, Musher DM, Niederman MS, Torres A, Whitney CG, Infectious Diseases Society of America; American Thoracic Society:
Infectious Diseases Society of America/American Thoracic Society Consensus guidelines on the management of community-acquired pneumonia in adults. Clin Infect Dis 2007, 44:S27-72.

14. American Thoracics Society/Infectious Diseases Society of America: Guidelines for management of adults with hospital-acquired, ventilatorassociated, and healthcare-associated pneumonia. Am J Respir Crit Care Med 2005, 171:388-416.

15. Solomkin JS, Mazuski JE, Bradley JS, Rodvold KA, Goldstein EJ, Baron EJ, O'Neill PJ, Chow AW, Dellinger EP, Eachempati SR, Gorbach S, Hilfiker M, May AK, Nathens AB, Sawyer RG, Bartlett JG: Diagnosis and management of complicated intra-abdominal infection in adults and children: guidelines by the Surgical Infection Society and the Infectious diseases Society of America. Clin Infect Dis 2010, 50:133-164.

16. Mermel LA, Allon M, Bouza E, Craven DE, Flynn P, O'Grady NP, Raad II, Rijnders BJ, Sherertz RJ, Warren DK: Clinical practice guidelines for the diagnosis and management of intravascular catheter-related infection: 2009 update by the Infectious Diseases Society of America. Clin Infect Dis 2009, 49:1-45

17. Gupta K, Hooton TM, Naber KG, Wullt B, Colgan R, Miller LG, Moran GJ, Nicolle LE, Raz R, Schaeffer AJ, Soper DE, Infectious Diseases Society of America; European Society for Microbiologyand Infectious Diseases: International clinical practice guidelines for the treatment of acute uncomplicated cystitis and pyelonephritis in women: a 2010 update by the Infectious diseases Society of America and the European Society for Microbiology and Infectious Diseases. Clin Infect Dis 2011, 52:e103-e120.

18. Stevens DL, Bisno AL, Chambers HF, Everett ED, Dellinger P, Goldstein EJ, Gorbach SL, Hirschmann JV, Kaplan EL, Montoya JG, Wade JC, Infectious Diseases Society of America: Practice guidelines for the diagnosis and management of skin and soft-tissue infections. Clin Infect Dis 2005, 41:1373-1406.

19. Corona A, Bertolini G, Ricotta AM, Wilson AP, Singer M: Variability of treatment duration for bacteraemia in the critically ill: a multinational survey. J Antimicrob Chemother 2003, 53:849-852.

20. Daneman N, Shore K, Pinto R, Fowler R: Antibiotic treatment duration for bloodstream Infections in critically ill patients: a national survey of Canadian infectious diseases and critical care specialists. Int J Antimicr Agents 2011, 38:480-485.

21. Kyriakidou KG, Rafailidis P, Matthaiou DK, Athanasiou S, Falagas ME: Shortversus long-course antibiotic therapy for acute pyelonephritis in adolescents and adults: a meta-analysis of randomized controlled trials. Clin Ther 2008, 30:1859-1868.

22. Dimopoulos G, Matthaiou DK, Karageorgopoulos DE, Grammatikos AP, Athanassa Z, Falagas ME: Short- versus long-course antibacterial therapy for community-acquired pneumonia: a meta-analysis. Drugs 2008 , 68:1841-1854

23. Li JZ, Winston LG, Moore DH, Bent S: Efficacy of short-course antibiotic regimens for community-acquired pneumonia: a meta-analysis. Am J Med 2007, 120:783-790.

24. Scottish Intercollegiate Guidelines Network: Randomised controlled trials search filter.[http://www.sign.ac.uk/methodology/filters.html].

25. Higgins JP, Thompson SG, Deeks JJ, Altman DG: Measuring inconsistency in meta-analysis. BMJ 2003, 327:557-560.

26. Chowdhary G, Dutta S, Narang A: Randomized controlled trial of 7-day vs. 14-day antibiotics for neonatal sepsis. J Trop Pediatr 2006, 52:427-432.

27. Basoli A, Chirletti P, Cirino E, D'Ovidio NG, Doglietto GB, Giglio D, Giulini SM Malizia A, Taffurelli M, Petrovic J, Ecari M, Italian Study Group: A prospective, double-blind, multicenter, randomized trial comparing ertapenem 3 vs $\geq 5$ days in community-acquired intraabdominal infection. J Gastrointest Surg 2008, 12:592-600.

28. Runyon BA, McHutchison JG, Antillon MR, Akriviadis EA, Montano AA: Short-course versus long-course antibiotic treatment of spontaneous bacterial peritonitis. Gastroenterology 1991, 100:1737-1742.

29. Chaudhry Zl, Nisar S, Ahmed U, et al: Short course of antibiotic treatment in spontaneous bacterial peritonitis: a randomized controlled study. JCPSP 2000, 10:284-288.

30. Engle WD, Jackson GL, Sendelbach DM, Stehel EK, Ford DM, McHugh KM, Norris MR, Vedro DA, Velaphi S, Michelow IC, Olsen KD: Pneumonia in term neonates: laboratory studies and duration of antibiotic therapy. $J$ Perinatol 2003, 23:372-377.

31. Engle WD, Jackson GL, Sendelbach D, Ford D, Olesen B, Burton KM, Pritchard MA, Frawley WH: Neonatal pneumonia: comparison of 4 vs 7 
days of antibiotic therapy in term and near-term infants. J Perinatol 2000, 20:421-426.

32. Vuori-Holopainen E, Peltola H, Kallio MJ, SE-TU Study Group: Narrowversus broad-spectrum parenteral antimicrobials against common infections of childhood: a prospective and randomised comparison between penicillin and cefuroxime. Eur J Pediatr 2000, 159:878-884.

33. Agarwal G, Awasthi S, Kabra SK, Kaul A, Singhi S, Walter SD, ISCAP Study Group: Three day versus five day treatment with amoxicillin for nonsevere pneumonia in young children: a multicentre randomised controlled trial. BMJ 2004, 328:791.

34. Pakistan Multicentre Amoxycillin Short Course Therapy (MASCOT) pneumonia study group: Clinical efficacy of 3 days versus 5 days of oral amoxicillin for treatment of childhood pneumonia: a multicentre double-blind trial. Lancet 2002, 360:835-841.

35. File TM Jr, Mandell LA, Tillotson G, Kostov K, Georgiev O: Gemifloxacin once daily for 5 days versus 7 days for the treatment of communityacquired pneumonia: a randomized, multicentre, double blind study. $J$ Antimicrob Chemother 2007, 60:112-120.

36. Tellier G, Niederman MS, Nusrat R, Patel M, Lavin B: Clinical and bacteriological efficacy and safety of 5 and 7 day regimens of telithromycin once daily compared with a 10 day regimen of clarithromycin twice daily in patients with mild to moderate community-acquired pneumonia. J Antimicrob Chemother 2004, 54:515-523.

37. Dunbar LM, Wunderink RG, Habib MP, Smith LG, Tennenberg AM, Khashab MM, Wiesinger BA, Xiang JX, Zadeikis N, Kahn JB: High-dose, short-course levofloxacin for community-acquired pneumonia: a new treatment paradigm. Clin Infect Dis 2003, 37:752-760.

38. Siegel RE, Alicea M, Lee A, Blaiklock R: Comparison of 7 versus 10 days of antibiotic therapy for hospitalized patients with uncomplicated community-acquired pneumonia: a prospective, randomized, double blind study. Am J Ther 1999, 6:217-222.

39. Leophonte $\mathrm{P}$, Choutet $\mathrm{P}$, Gaillat J, et al: Efficacy of a ten day course of ceftriaxone compared to a shortened five day course in the treatment of community-acquired pneumonia in hospitalized adults with risk factors. Medecine et Maladies Infectieuses 2002, 32:369-381.

40. el Moussaoui $R$, de Borgie CA, van den Broek P, Hustinx WN, Bresser $P$, van den Berk GE, Poley JW, van den Berg B, Krouwels FH, Bonten MJ, Weenink C, Bossuyt PM, Speelman P, Opmeer BC, Prins JM: Effectiveness of discontinuing antibiotic treatment after three days versus eight days in mild to moderate-severe community acquired pneumonia: randomised, double blind study. BMJ 2006, 332:1355-1360

41. Fekih Hassen M, Ayed S, Ben Sik Ali H, Gharbi R, Marghli S, Elatrous S: Duration of antibiotic therapy for ventilator-associated pneumonia: comparison of 7 and 10 days. Ann Fr Anesth Reanim 2009, 28:16-23.

42. Chastre J, Wolff M, Fagon JY, Chevret S, Thomas F, Wermert D, Clementi E, Gonzalez J, Jusserand D, Asfar P, Perrin D, Fieux F, Aubas S, PneumA TrialGroup: Comparison of 8 vs 15 days of antibiotic therapy for ventilator-associated pneumonia in adults: a randomized trial. JAMA 2003, 290:2588-2598.

43. de Gier R, Karperien A, Bouter K, Zwinkels M, Verhoef J, Knol W, Boon T, Hoepelman IM: A sequential study of intravenous and oral fleroxacin for 7 or 14 days in the treatment of complicated urinary tract infections. Int J Antimicrob Agents 1995, 6:27-30

44. Stamm WE, McKevitt M, Counts GW: Acute renal infection in women: treatment with trimethoprim-sulfamethoxazole or ampicillin for two or six weeks. Ann Intern Med 1987, 106:341.

45. Gleckman R, Bradley P, Roth R, Hibert D, Pelletier C: Therapy of symptomatic pyelonephritis in women. J Urol 1985, 133:176-178.

46. Pylkkanen J, Vilska J, Koskimies O: The length of antimicrobial therapy in lower vs. upper urinary tract infection of childhood. Acta Paediatr Scand 1981, 70:885-888.

47. Jernelius H, Zbornik J, Bauer CA: One or three weeks' treatment of acute pyelonephritis? A double-blind comparison, using a fixed combination of pivampicillin plus pivmecillinam. Acta Med Scand 1988, 223:469-477.

48. Cheng C, Tsau Y, Lin T: Effective duration of antimicrobial therapy for the treatment of acute lobar nephronia. Pediatrics 2006, 117:e84-e89.

49. Hepburn MJ, Dooley DP, Skidmore PJ, Ellis MW, Starnes WF, Hasewinkle WC: Comparison of short-course ( 5 days) and standard (10 days) treatment for uncomplicated cellulitis. Arch Intern Med 2004, 164:1669-1674.
50. Liu C, Bayer A, Cosgrove SE, et al: Clinical practice guidelines by the Infectious Diseases Society of America for the treatment of Methicillinresistant Staphylococcus aureus infections in adults and children. Clin Infect Dis 2011, 52:1-38.

51. Jernigan JA, Farr BM: Short-course therapy of catheter-related Staphylococcus aureus bacteremia: a meta-analysis. Ann Intern Med 1993 119:304-311.

52. Ehni WF, Reller LB: Short-course therapy for catheter-associated Staphylococcus aureus bacteremia. Arch Intern Med 1989, 149:533-536.

53. Hedrick TL, Evans HL, Smith RL, McElearney ST, Schulman AS, Chong TW, Pruett TL, Sawyer RG: Can we define an ideal duration of antibiotic therapy? Surg Infect 2006, 7:419-432.

54. Scalera NM, File TM: How long should we treat community-acquired pneumonia? Curr Opin Infect Dis 2007, 20:177-181.

55. File TM: Clinical efficacy of newer agents in short-duration therapy for community-acquired pneumonia. Clin Infect Dis 2004, 39:S159-164.

56. Klausner HA, Brown P, Peterson J, Kaul S, Khashab M, Fisher AC, Kahn JB: A trial of levofloxacin $750 \mathrm{mg}$ once daily for 5 days versus ciprofloxacin $400 \mathrm{mg}$ and/or $500 \mathrm{mg}$ twice daily for 10 days in the treatment of acute pyelonephritis. Curr Med Res Opin 2007, 23:2637-2645.

57. Peterson J, Kaul S, Khashab M, Fisher AC, Kahn JB: A double-blind, randomized comparison of levofloxacin $750 \mathrm{mg}$ once-daily for five days with ciprofloxacin $400 / 500 \mathrm{mg}$ twice-daily for 10 days for the treatment of complicated urinary tract infections and acute pyelonephritis. Urology 2008, 71:17-22

58. Talan DA, Stamm WE, Hooton TM, Moran GJ, Burke T, Iravani A, ReuningScherer J, Church DA: Comparison of ciprofloxacin (7 days) and trimethoprim-sulfamethoxazole (14 days) for acute uncomplicated pyelonephritis in women: a randomized trial. JAMA 2000, 283:1583-1590.

doi:10.1186/cc10545

Cite this article as: Havey et al:: Duration of antibiotic therapy for bacteremia: a systematic review and meta-analysis. Critical Care 2011 15: R267.

\section{Submit your next manuscript to BioMed Central and take full advantage of:}

- Convenient online submission

- Thorough peer review

- No space constraints or color figure charges

- Immediate publication on acceptance

- Inclusion in PubMed, CAS, Scopus and Google Scholar

- Research which is freely available for redistribution 